\title{
Modeling, Identification, and Feedback Control of Noise in an Acoustic Duct
}

\author{
Jeongho Hong, James C. Akers, Ravinder Venugopal, Miin-Nan Lee, \\ Andrew G. Sparks, Peter D. Washabaugh, and Dennis S. Bernstein
}

\begin{abstract}
Although active noise control has been a subject of interest for over 50 years, it has become feasible only with recent technological advances. This paper formulates the problem of noise control in a one-dimensional acoustic duct in a form that lends itself to the application of feedback control theory. In contrast to most of the literature on the subject which uses feedforward techniques, a feedback approach is used. Inconsistencies that appear in previous feedback control models are rectified, controllers are designed using precompensated linear quadratic Gaussian (LQG) synthesis, and experimental verification of the control designs is presented. The experimental results show a reduction of about 5-12 dB over a frequency range from 150-350 Hz.
\end{abstract}

\section{INTRODUCTION}

A LTHOUGH active suppression of noise has been a technological objective for more than half of a century [1]-[3], it is only recently with the advent of low-cost highspeed processors that this objective has become feasible. The proven and potential applications of active noise suppression are vast, ranging from aircraft engines to automobile interiors to heating, ventilation, and air conditioning (HVAC) systems to household appliances. The broad applicability, technological feasibility, and nonhazardous nature of active noise control technology represent a major opportunity for control engineering.

The most widely used approach to active noise control technology is based upon adaptive disturbance cancellation techniques [3]-[5]. These techniques involve a gradient-type search for control gains to reject undesirable disturbances. Because these methods require disturbance measurements, they are traditionally viewed as feedforward algorithms.

An alternative approach to the active noise control problem is to apply standard feedback control techniques. Although such techniques have been widely applied throughout almost all areas of engineering, they have seen relatively little application in the area of active noise control. Exceptions include the work of Radcliffe and co-workers [6]-[8], who demonstrated the feasibility of applying feedback control to the problem of suppressing noise in a one-dimensional duct with circular cross section, and Yang et al. [9] who used robust feedback control design techniques to attenuate structurally generated

Manuscript received October 13, 1994. Recommended by Associate Editor, E. G. Collins, Jr. This work was supported in part by the Air Force Office of Scientific Research under Grants F49620-92-J-0127 and F49620-95-1-0019.

The authors are with the Department of Aerospace Engineering, University of Michigan, Ann Arbor, MI 48109-2118 USA.

Publisher Item Identifier S 1063-6536(96)03345-3. interior noise in a reverberant acoustic enclosure. Recently, researchers have explored the relationship between feedback control techniques and feedforward methods [10] and [11].

The goal of the present paper is to continue the investigation of [8] into feedback control techniques for active noise suppression. Since our primary motivation is noise suppression in air conditioning systems, we consider a duct with rectangular cross section. We begin our development with a detailed derivation of the state-space model of a duct with speaker and microphone. Although portions of this derivation are largely standard (see [12, (4-3.7)], [13], and [14]), this derivation serves several purposes. First, we correct a dimensional inconsistency that can be found in $[8,(1)]$ and which can be traced to the dimensions ascribed to the spatial delta function. These inconsistencies arise from the fact that the derivation in [12]-[14] and related work applies to the three-dimensional case, while the specialization to one dimension is not immediate. And, second, this development provides a largely self-contained derivation of the dynamic equations in a recognizable form that is accessible to control engineers accustomed to state-space models that are required for modern feedback synthesis. Finally, this derivation accounts for all of the nonlinear terms that arise and illustrates the meaning of each physical parameter and its role in the plant dynamics.

In the present paper we consider a single-input, singleoutput plant involving one control actuator (speaker) and one control sensor (microphone). Additional speakers and microphones are used to provide disturbances and to assess closed-loop performance. To simplify matters, we confine our consideration in this paper to the case of a colocated sensor and actuator, that is, the control speaker and control microphone located at the same position along the duct. This configuration has been studied in the noise control literature under the name of tightly coupled monopole [15]-[17]. In contrast to feedforward control, however, we do not assume that a measurement of the disturbance is available.

In designing feedback controllers for the acoustic duct, we apply modern state-space control techniques. The use of such techniques is necessitated by the high order of the identified model, which, for a $400 \mathrm{~Hz}$ modeling bandwidth in our experiment, involves 30 states. Feedback controllers designed for noise suppression were obtained by applying linear quadratic Gaussian (LQG) synthesis with suitable precompensation [18] to assure robustness to high-frequency uncertainty. Experimental results that show significant noise reduction over a broad frequency range are presented. 


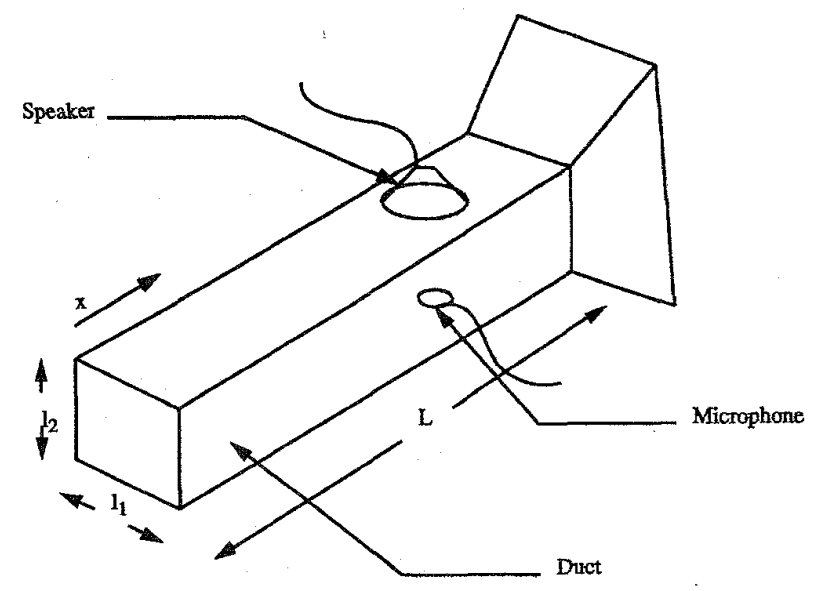

Fig. 1. Acoustic duct.

\section{State-Space Model of the ACOỦstic DUCT WITH SENSOR AND ACTUATOR}

The duct is assumed to be rectangular in cross section and open at both ends. A fairing is attached to one end to accommodate a fan for future investigation of the effects of mean flow and fan noise. The dimensions $l_{1}$ and $l_{2}$ of the duct are sufficiently small compared to its length $L\left(l_{1} / L, l_{2} / L \ll\right.$ 1) that acoustic waves travel along the axis of the duct with planar wave fronts. This assumption enables us to treat the duct as a one-dimensional waveguide with spatial coordinate $x$, where $0 \leq x \leq L$. The control actuator is a speaker located in the wall of the duct in its plane at $x=x_{s}$, while the sensor is a microphone placed inside the duct at the same location, as depicted in Fig. 1.

The state-space model of the acoustic duct with actuating speaker in the duct wall and sensing microphone is developed from three fundamental equations that characterize the propagation of an acoustic disturbance through a fluid. The first of these equations is the equation of state ([14, pp. 100-101])

$$
p(x, t)=c^{2} \rho(x, t) S(x, t)
$$

where $p(x, t)$ is the acoustic pressure, $\rho(x, t)$ is the density, $c=\sqrt{\mathcal{B} / \rho_{0}}$ is the phase speed of the acoustic wave $(343 \mathrm{~m} / \mathrm{s}$ in air at room conditions), $\mathcal{B}$ is the adiabatic bulk modulus of the medium ( $1.423 \times 10^{5} \mathrm{~Pa}$ for air at room conditions), $\rho_{0}$ is the equilibrium density $\left(1.21 \mathrm{~kg} / \mathrm{m}^{3}\right.$ for air at room conditions), and $S(x, t) \triangleq\left[\rho(x, t)-\rho_{0}\right] / \rho_{0}$ is the condensation of the fluid. The acoustic pressure $p(x, t)$ is related to the absolute pressure $P(x, t)$ and the equilibrium pressure $P_{0}$ by $p(x, t)=$ $P(x, t)-P_{0}$.

The second fundamental equation is the equation of continuity. To derive this equation with the speaker as a point mass source or sink, consider the infinitesimal volume element $\Delta x \Delta y \Delta z$. shown in Fig. 2. The mass flux $M_{\text {left }}$ entering through the left side of the volume element, assuming that the fluid particle velocity $v(x, t)$ is in the positive $x$ direction, is given by

$$
M_{\text {left }}=\rho(x, t) v(x, t) \Delta y \Delta z
$$

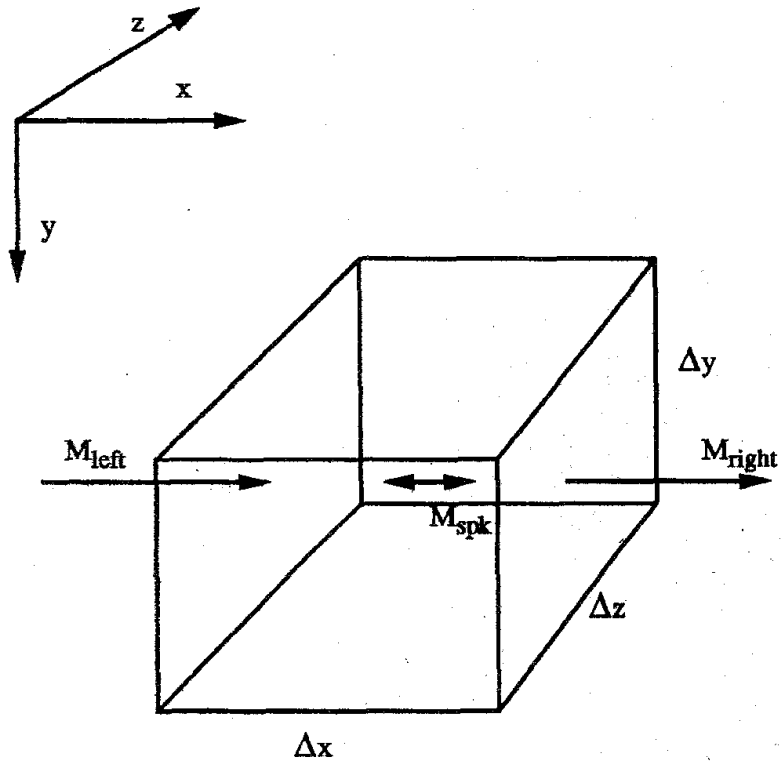

Fig. 2. Volume element in acoustic medium.

while the mass flux $M_{\text {right }}$ exiting through the right side of the element in the $x$ direction is

$$
\begin{aligned}
M_{\text {right }}= & \left\{\rho(x, t) v(x, t)+[\rho(x, t) v(x, t)]_{x} \Delta x\right\} \\
& \cdot \Delta y \Delta z
\end{aligned}
$$

Suppose the speaker is a piston with cross-sectional area $A_{s}$, which injects mass into the duct in the $x$ direction with velocity $v_{s}(x, t)$. The speaker is assumed to be an infinitesimal source of length $\Delta x$ located at $x_{s}$ so that

$$
\begin{aligned}
v_{s}(x, t) & =0, & & 0 \leq x<x_{s}, \\
& =v_{s}(t), & & x_{s} \leq x \leq x_{s}+\Delta x, \\
& =0, & & x_{s}+\Delta x<x \leq L
\end{aligned}
$$

where $v_{s}(t)$ is the speaker baffle velocity. The mass per unit time injected into the duct by the speaker is $\rho(x, t) v_{s}(x, t) A_{s}$, and the equivalent mass flux $M_{s}$ into the volume element is given by

$$
M_{s}=\frac{\rho(x, t) v_{s}(x, t) A_{s}}{A_{s}} \Delta y \Delta z .
$$

The total increase in mass in the volume element per unit time is

$$
\begin{aligned}
M_{\text {left }}-M_{\text {right }}+M_{s}= & -[\rho(x, t) v(x, t)]_{x} \Delta x \Delta y \Delta z \\
& +\rho(x, t) v_{s}(x, t) \Delta y \Delta z
\end{aligned}
$$

Since the element does not move with the medium, however, the mass increase of the volume element per unit time is the rate of change of density within the element multiplied by the volume of the element, that is

$$
M_{\text {left }}-M_{\text {right }}+M_{s}=\rho_{t}(x, t) \Delta x \Delta y \Delta z .
$$

From (6) and (7) it follows that

$$
\begin{aligned}
\rho_{t}(x, t) \Delta x \Delta y \Delta z=- & {[\rho(x, t) v(x, t)]_{x} \Delta x \Delta y \Delta z } \\
& +\rho(x, t) v_{s}(x, t) \Delta y \Delta z
\end{aligned}
$$


which implies that

$$
\rho_{t}(x, t)=-[\rho(x, t) v(x, t)]_{x}+\frac{\rho(x, t) v_{s}(x, t)}{\Delta x} .
$$

Taking the speaker to be a point source, let $\Delta x \rightarrow 0$. If $x_{a} \leq x_{s}<x_{b}$, it follows from (4) that

$$
\begin{aligned}
\lim _{\Delta x \rightarrow 0} \int_{x_{a}}^{x_{b}} \frac{v_{s}(x, t)}{\Delta x} d x & =\lim _{\Delta x \rightarrow 0} \int_{x_{s}}^{x_{s}+\Delta x} \frac{v_{s}(x, t)}{\Delta x} d x \\
& =\lim _{\Delta x \rightarrow 0} v_{s}(t) \int_{x_{s}}^{x_{s}+\Delta x} \frac{1}{\Delta x} d x \\
& =v_{s}(t)
\end{aligned}
$$

Therefore

$$
\lim _{\Delta x \rightarrow 0} \frac{v_{s}(x, t)}{\Delta x}=v_{s}(t) \delta\left(x-x_{s}\right)
$$

where $\delta\left(x-x_{s}\right)$ is the spatial delta function concentrated at $x=x_{s}$ with dimensions (length) ${ }^{-1}$. Hence, we can rewrite (9) as

$$
\rho_{t}(x, t)=-[\rho(x, t) v(x, t)]_{x}+M(x, t)
$$

where

$$
M(x, t) \triangleq \rho(x, t) v_{s}(t) \delta\left(x-x_{s}\right) .
$$

The third fundamental equation is the linearized inviscid force equation derived from Euler's equation [14, pp. 102-104]

$$
p_{x}(x, t)=-\rho_{0} v_{t}(x, t) .
$$

Differentiating this equation with respect to $x$ yields

$$
-\rho_{0} v_{x t}(x, t)=p_{x x}(x, t) .
$$

Next, substituting $\rho(x, t)=\rho_{0}[1+S(x, t)]$ in the equation of state (1) and differentiating twice with respect to $t$ implies that

$$
[1+2 S(x, t)] \rho_{0} S_{t t}(x, t)=\frac{1}{c^{2}} p_{t t}(x, t)-2 \rho_{0} S_{t}^{2}(x, t) .
$$

Substituting $\rho(x, t)=\rho_{0}[1+S(x, t)]$ in (12) yields

$$
\begin{aligned}
\rho_{0} S_{t}(x, t)= & -\rho_{0}\left\{S_{x}(x, t) v(x, t)+[1+S(x, t)] v_{x}(x, t)\right\} \\
& +M(x, t)
\end{aligned}
$$

while differentiating (17) with respect to $t$ implies

$$
\begin{aligned}
\rho_{0} S_{t t}(x, t)= & -\rho_{0}\left\{S_{x t}(x, t) v(x, t)+S_{x}(x, t) v_{t}(x, t)\right. \\
& \left.+S_{t}(x, t) v_{x}(x, t)+[1+S(x, t)] v_{x t}(x, t)\right\} \\
& +M_{t}(x, t) .
\end{aligned}
$$

From (15), (16), and (18) we obtain

$$
\begin{aligned}
\frac{1}{c^{2}} p_{t t}(x, t)= & p_{x x}(x, t)+N(x, t) \\
& +[1+2 S(x, t)] M_{t}(x, t)
\end{aligned}
$$

where

$$
\begin{aligned}
N(x, t) \triangleq & 2 \rho_{0}^{2} S_{t}^{2}(x, t)+2 S(x, t) p_{x x}(x, t) \\
& -\rho_{0}[1+2 S(x, t)] \\
& \cdot\left[S_{x t}(x, t) v(x, t)+S_{x}(x, t) v_{t}(x, t)\right. \\
& \left.+S_{t}(x, t) v_{x}(x, t)+S(x, t) v_{x t}(x, t)\right]
\end{aligned}
$$

Differentiating (13) with respect to time and substituting $M_{t}(x, t)$ into (19) yields

$$
\begin{aligned}
\frac{1}{c^{2}} p_{t t}(x, t)= & p_{x x}(x, t)+\rho_{0} \dot{v}_{s}(t) \delta\left(x-x_{s}\right) \\
& +N(x, t)+N_{s}(x, t)
\end{aligned}
$$

where

$$
\begin{aligned}
N_{s}(x, t) \triangleq & \rho_{0}\left[S_{t}(x, t) v_{s}(x, t)+S(x, t) v_{s}(t)\right] \\
& \cdot \delta\left(x-x_{s}\right) .
\end{aligned}
$$

Note that $N(x, t)$ and $N_{s}(x, t)$ contain all of the nonlinear terms in (21). Deleting these terms yields the linearized nonhomogeneous equation

$$
\frac{1}{c^{2}} p_{t t}(x, t)=p_{x x}(x, t)+\rho_{0} \dot{v}_{s}(t) \delta\left(x-x_{s}\right)
$$

which describes the pressure in the acoustic duct with the speaker attached to the duct wall.

Note that (23) is dimensionally consistent. In contrast, [8, (1)], which involves particle displacement and an end speaker, is not dimensionally consistent. Specifically, with the correct dimensions attributed to the spatial delta function, $[8,(1)]$ has an additional dimension of length on the left-hand side.

Using separation of variables, let

$$
p(x, t)=q(t) V(x)
$$

and consider the homogeneous equation

$$
\frac{1}{c^{2}} p_{t t}(x, t)=p_{x x}(x, t) \text {. }
$$

Substituting (24) into (25) and rearranging gives

$$
V_{x x}(x)+\frac{\lambda}{c^{2}} V(x)=0
$$

where $\lambda$ is a positive constant. Hence

$$
V(x)=\alpha \sin k x+\beta \cos k x
$$

where $k^{2} \triangleq \lambda / c^{2}$. From the open-open boundary conditions $p(0, t)=p(L, t)=0$, it follows that

$$
\begin{aligned}
V(0) & =\beta \\
& =0, \\
V(L) & =\alpha \sin k L \\
& =0 .
\end{aligned}
$$

Therefore, $k=k_{i} \triangleq i \pi / L, i=1,2,3, \cdots$. We define $\omega_{n i} \triangleq k_{i} c$, which gives $V_{i}(x)=\alpha \sin k_{i} x$. For convenience, $V_{i}(\cdot)$ is scaled so that

$$
\begin{aligned}
\left\langle V_{i}(\cdot), \frac{1}{c^{2}} V_{j}(\cdot)\right\rangle & \triangleq \frac{1}{c^{2}} \int_{0}^{L} V_{i}(x) V_{j}(x) d x \\
& =\delta_{i j}
\end{aligned}
$$

where $\delta_{i j}$ is the Kronecker delta. This implies that $\alpha=$ $c \sqrt{2 / L}$ and thus

$$
V_{i}(x)=c \sqrt{\frac{2}{L}} \sin k_{i} x, \quad i=1,2,3, \cdots
$$


Returning to the nonhomogeneous equation (23), let

$$
p(x, t)=\sum_{j=1}^{\infty} q_{j}(t) V_{j}(x)
$$

so that

$$
\frac{1}{c^{2}} \sum_{j=1}^{\infty}\left[\ddot{q}_{j}(t)+\lambda_{j} q_{j}(t)\right] V_{j}(x)=\rho_{0} \dot{v}_{s}(t) \delta\left(x-x_{s}\right) .
$$

Taking the inner product of both sides of (32) with $V_{i}(\cdot)$ as in $(28)$ yields

$$
\ddot{q}_{i}(t)+\lambda_{i} q_{i}(t)=b_{i} u_{s}(t), \quad i=1,2,3, \cdots
$$

where $b_{i} \triangleq V_{i}\left(x_{s}\right)$ and $u_{s}(t) \triangleq \rho_{0} \dot{v}_{s}(t)$.

To obtain a state-space form, we consider $r$ modes

$$
\begin{aligned}
q(t) & \triangleq\left[\begin{array}{lll}
q_{1}(t) & \cdots & q_{r}(t)
\end{array}\right]^{T}, \\
B_{0} & \triangleq\left[\begin{array}{lll}
b_{1} & \cdots & b_{r}
\end{array}\right]^{T}
\end{aligned}
$$

and rewrite (33) in the form

$$
\ddot{q}(t)+\Omega^{2} q(t)=B_{0} u_{s}(t)
$$

where $\Omega \triangleq \operatorname{diag}\left(\omega_{n 1}, \cdots, \omega_{n r}\right)$. Equation (35) is the undamped normal mode equation for the acoustic duct with actuation.

For measurement we consider two types of microphones, namely, pressure type and pressure gradient type. For the case of a pressure microphone located at $x=x_{m}$, the measurement $y_{m}(t)$ is given by

$$
\begin{aligned}
y_{m}(t) & =p\left(x_{m}, t\right) \\
& =\sum_{j=1}^{\infty} q_{j}(t) V_{j}\left(x_{m}\right)
\end{aligned}
$$

which, retaining $r$ modes, can be written as

$$
y_{m}(t)=C_{0} q(t)
$$

where

$$
\dot{C}_{0} \triangleq\left[\begin{array}{lll}
V_{1}\left(x_{m}\right) & \cdots & V_{r}\left(x_{m}\right)
\end{array}\right] .
$$

For the case of a pressure gradient microphone located at $x=x_{m}$, the measurement is

$$
\begin{aligned}
y_{m}(t) & =p_{x}\left(x_{m}, t\right) \\
& =\sum_{i=1}^{\infty} q_{i}(t) V_{i x}\left(x_{m}\right)
\end{aligned}
$$

which, retaining $r$ modes, can be written as (37) with

$$
C_{0} \triangleq\left[\begin{array}{lll}
V_{1 x}\left(x_{m}\right) & \cdots & V_{r x}\left(x_{m}\right)
\end{array}\right]
$$

The microphone provides an output voltage $y(t)$ that depends on the measured pressure or pressure gradient. Assuming that the transfer function of this device is a pure gain $K_{m}$, we obtain

$$
y(t)=K_{m} y_{m}(t)
$$

To obtain a state-space description of the acoustic duct, let

$$
x_{d}(t) \triangleq\left[\begin{array}{lllll}
q_{1}(t) & \dot{q}_{1}(t) & \cdots & q_{r}(t) & \dot{q}_{r}(t)
\end{array}\right]^{T}
$$

so that

$$
\begin{aligned}
& \dot{x}_{d}(t)=A_{d} x_{d}(t)+B_{d} u_{s}(t), \\
& y_{d}(t)=C_{d} x_{d}(t)
\end{aligned}
$$

where, with the inclusion of proportional damping and assuming that a pressure-type microphone is used

$$
\begin{aligned}
& A_{d} \triangleq \text { block-diag }\left(\left[\begin{array}{cc}
0 & 1 \\
-\omega_{n 1}^{2} & -2 \zeta_{1} \omega_{n 1}
\end{array}\right], \cdots,\right. \\
& \left.\left[\begin{array}{cc}
0 & 1 \\
-\omega_{n r}^{2} & -2 \zeta_{r} \omega_{n r}
\end{array}\right]\right) \\
& B_{d} \triangleq\left[\begin{array}{lllll}
0 & b_{1} & \cdots & 0 & b_{r}
\end{array}\right]^{T}, \\
& C_{d} \triangleq\left[\begin{array}{lllll}
V_{1}\left(x_{m}\right) & 0 & \ldots & V_{r}\left(x_{m}\right) & 0
\end{array}\right] .
\end{aligned}
$$

Next, we incorporate a model of the speaker in the statespace description. The transfer function from the speaker voltage input $V_{s}$ to the speaker baffle acceleration $\dot{v}_{s}$ is given by

$$
\frac{s v_{s}(s)}{V_{s}(s)}=\frac{K_{s} s^{2}}{s^{2}+2 \zeta_{s} \omega_{n s} s+\omega_{n s}^{2}}
$$

where $K_{s}$ is a speaker constant, $\omega_{n s}$ is the natural frequency of the speaker, and $\zeta_{s}$ is the damping ratio. Let the state-space realization of this transfer function be given by

$$
\begin{aligned}
& \dot{x}_{s}(t)=A_{s} x_{s}(t)+B_{s} V_{s}(t), \\
& \dot{v}_{s}(t)=C_{s} x_{s}(t)+D_{s} V_{s}(t)
\end{aligned}
$$

where

$$
\begin{aligned}
& A_{s} \triangleq\left[\begin{array}{cc}
0 & 1 \\
-\omega_{n s}^{2} & -2 \zeta_{s} \omega_{n s}
\end{array}\right] \text {, } \\
& B_{s} \triangleq\left[\begin{array}{c}
0 \\
K_{s}
\end{array}\right], \\
& C_{s} \triangleq\left[\begin{array}{ll}
-\omega_{n s}^{2} & -2 \zeta_{s} \omega_{n s}
\end{array}\right] \text {, } \\
& D_{s} \triangleq K_{s} \text {. }
\end{aligned}
$$

Defining the augmented state vector

$$
x(t) \triangleq\left[\begin{array}{l}
x_{s}(t) \\
x_{d}(t)
\end{array}\right]
$$

(43), (44), (49), and (50) can be combined as

$$
\begin{aligned}
& \dot{x}(t)=A x(t)+B u(t), \\
& y(t)=C x(t)
\end{aligned}
$$

where

$$
\begin{aligned}
A & \triangleq\left[\begin{array}{cc}
A_{s} & 0 \\
\rho_{0} B_{d} C_{s} & A_{d}
\end{array}\right], \\
B & \triangleq\left[\begin{array}{c}
B_{s} \\
\rho_{0} D_{s} B_{d}
\end{array}\right], \\
C & \triangleq\left[\begin{array}{ll}
0 & K_{m} C_{d}
\end{array}\right], \\
u(t) & \triangleq V_{s}(t) .
\end{aligned}
$$

Note that (54) has order $n_{\text {plant }}=2 r+2$. 


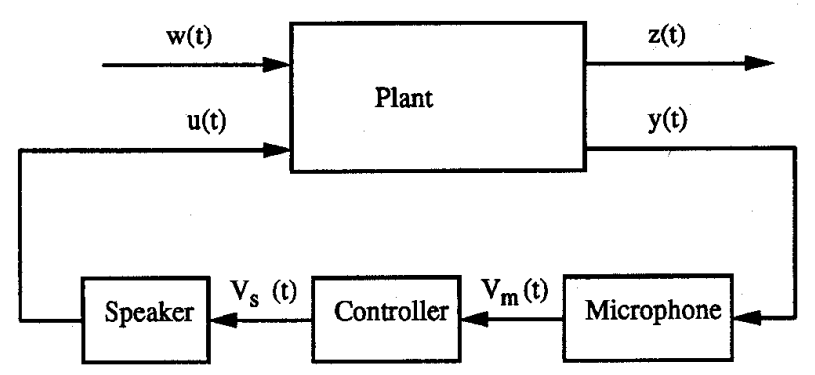

Fig. 3. Closed-loop system.

The control objective is to minimize the acoustic pressure due to the disturbance input at a specified performance location $x=x_{p}$. In the closed-loop configuration, the input to the controller is the output voltage from the microphone, while its output is a commanded voltage that drives the actuating speaker.

For controller synthesis, a disturbance noise source located at $x=x_{d}$ is introduced and sensor noise is included in the model. The plant shown in Fig. 3 thus has the state-space representation

$$
\begin{aligned}
& \dot{x}(t)=A x(t)+B u(t)+D_{1} w(t), \\
& y(t)=C x(t)+D_{2} w(t)
\end{aligned}
$$

where $D_{1}$ is given by

$$
D_{1} \triangleq\left[\begin{array}{ccccccc}
0 & 0 & 0 & V_{1}\left(x_{d}\right) & \cdots & 0 & V_{r}\left(x_{d}\right) \\
0 & 0 & 0 & 0 & \cdots & 0 & 0
\end{array}\right]^{T}
$$

and where the matrix $D_{2}=\left[\begin{array}{ll}0 & D_{20}\end{array}\right]$ represents sensor noise. The vector $w(t)$ is defined as

$$
w(t) \triangleq\left[\begin{array}{l}
w_{1}(t) \\
w_{2}(t)
\end{array}\right]
$$

where $w_{1}(t)$ is the disturbance signal and $w_{2}(t)$ is sensor noise. The signals $w_{1}(t)$ and $w_{2}(t)$ are assumed to be uncorrelated unit-intensity white noise processes.

The performance variable $z(t)$ is a weighted sum of the states and the control input vector, that is

$$
z(t)=E_{1} x(t)+E_{2} u(t)
$$

Since the control objective is to minimize the acoustic pressure at a given performance point, the matrix $E_{1}$ has the same form as the matrix $C$ for a pressure type microphone and is thus given by

$$
E_{1}=\left[\begin{array}{lllllll}
0 & 0 & \varepsilon_{1} V_{1}\left(x_{p}\right) & 0 & \cdots & \varepsilon_{r} V_{r}\left(x_{p}\right) & 0
\end{array}\right]
$$

where $x_{p}$ is the point at which performance is to be optimized, and $\varepsilon_{1}, \cdots, \varepsilon_{r}$ are weights that can be chosen as design parameters. Equations (58), (59), and (62) represent the plant with sensor and actuator in a form suitable for control design.
TABLE I

Modal Freouencies and Damping Ratios of THE Duct IDentified with Disturbance Speaker

\begin{tabular}{|c|c|c|c|c|}
\hline Mode & $f_{\text {ni }}(\mathrm{Hz})$ (Exp.) & $f_{\mathrm{ni}}(\mathrm{Hz})$ (Theory) & \% Error & $\zeta_{i}$ \\
\hline 1 & 125.56 & 128.00 & $1.94 \%$ & $6.43 \%$ \\
2 & 179.27 & 170.67 & $4.79 \%$ & $7.11 \%$ \\
3 & 221.37 & 213.33 & $3.63 \%$ & $2.35 \%$ \\
4 & 269.97 & 256.00 & $5.17 \%$ & $4.95 \%$ \\
5 & 321.32 & 298.67 & $7.05 \%$ & $4.23 \%$ \\
6 & 352.90 & 341.33 & $3.27 \%$ & $1.63 \%$ \\
7 & 384.89 & 384.00 & $0.23 \%$ & $1.56 \%$ \\
\hline
\end{tabular}

\section{IDENTIFICATION OF DUCT, SPEAKER, AND MICROPHONE PARAMETERS}

The parameters of the state-space model of the duct described in the previous section are obtained by experimental identification. Although many of these parameters, such as the modal frequencies, can be estimated from the theoretical development, others, such as the damping ratios, must be experimentally identified. The transfer function (48) of the speaker from input voltage to speaker baffle acceleration is obtained by first determining the transfer function from input voltage to speaker baffle displacement. This is done by measuring the baffle displacement in response to a known input voltage using a photonic sensor. The acceleration of the baffle is then evaluated as the second time derivative of its displacement. The modal frequency $f_{n s}=\omega_{n s} / 2 \pi$ and the damping ratio $\zeta_{n s}$ of the speaker are found to be $67 \mathrm{~Hz}$ and $74 \%$, respectively.

The duct is $12 \mathrm{ft}$ in length and has cross-section 8 in $x$ 12 in. The fairing that is attached at one end is $2 \mathrm{ft}$ long. The duct parameters are identified by introducing a known signal consisting of 400 simultaneous sinusoidal tones of various frequencies through either the control or the disturbance speaker, and using a spectrum analyzer to perform a fast Fourier transform (FFT) on the microphone voltage output. Since the loudspeakers have poor frequency response below $100 \mathrm{~Hz}$ and the type of acoustic disturbance in this study is chosen to have frequency components limited to the $400 \mathrm{~Hz}$ range, the system is identified from 100 to $400 \mathrm{~Hz}$.

A least-squares curve fit is performed to determine a transfer function that matches the gain and phase of the experimental frequency response, and the modal frequencies and damping ratios are determined from this transfer function. With this method, a transfer function from speaker voltage input $u(t)$ to microphone voltage output $y(t)$, corresponding to (54) and (55), is obtained in one step.

The theoretical and identified natural frequencies and identified damping ratios of the modes of the duct are given in Tables I and II, while experimental and identified modal frequency response plots of the duct for disturbance source and control speaker are shown in Figs. 4 and 5, respectively. Table I also lists the theoretical estimates of the modal frequencies. The first cross mode has a frequency above $450 \mathrm{~Hz}$, and for the amplitudes of the acoustic disturbances used, the walls of the duct remain rigid. Hence, these aspects, as well as the effect of duct termination impedances, do not seem to affect the model to any considerable degree. The correlation 
TABLE II

MODAL FREQUENCIES AND DAMPING RATIOS OF the Duct IDENTIFIEd WITH CONTROL SPEAKER

\begin{tabular}{|c|c|c|}
\hline Mode & $f_{\mathrm{ni}}(\mathrm{Hz})$ & $\zeta_{i}$ \\
\hline 1 & 123.29 & $18.30 \%$ \\
2 & 141.47 & $8.82 \%$ \\
3 & 182.65 & $5.41 \%$ \\
4 & 228.30 & $3.37 \%$ \\
5 & 262.78 & $5.28 \%$ \\
6 & 314.06 & $3.69 \%$ \\
7 & 340.45 & $2.46 \%$ \\
8 & 366.46 & $3.51 \%$ \\
\hline
\end{tabular}
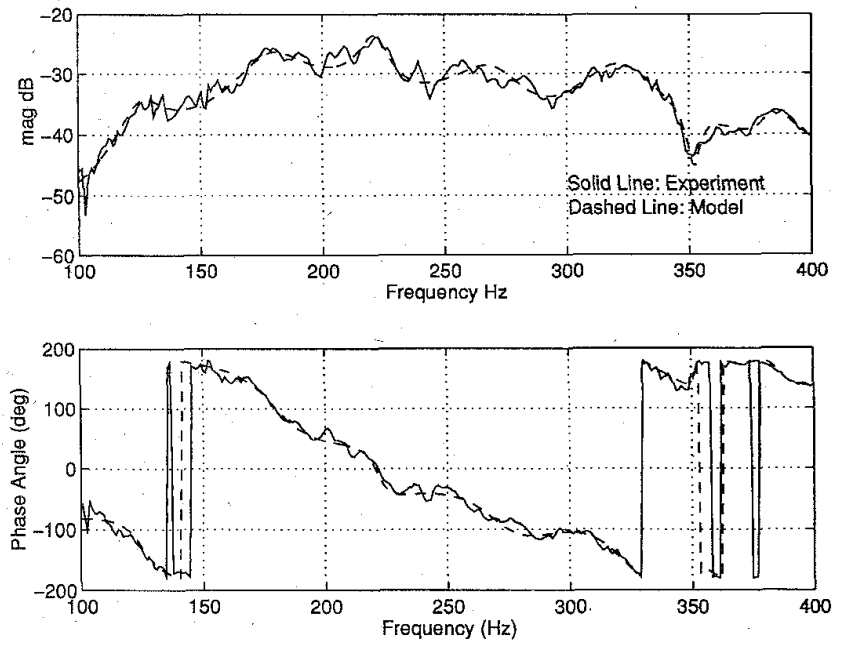

Fig. 4. Frequency response of the duct from disturbance speaker to microphone.

between the theoretical and experimental modal frequencies suggests that finite speaker size does not have a significant effect, Pole-zero maps for the identified models of the duct with disturbance speaker and control speaker are shown in Figs. 6 and 7. By combining the models identified using the disturbance and control speakers, we obtain an identified model of the duct with $r=15$ modes in the frequency range of 100 to $450 \mathrm{~Hz}$.

One factor to be noted in the identification of the duct is that, at least theoretically, the modes identified using the control speaker and the disturbance speaker should be identical. As can be seen from Tables I and II, however, some of the modes observed while using the control speaker are not observed while using the disturbance speaker. Also, there are small discrepancies in the natural frequencies of the modes that are common to both identified transfer functions with more distinct differences in the damping ratios of these modes. These variations can be attributed to the following factors. First, the control and disturbance speakers are not identical, specifically, the control speaker is an 8 -in woofer while the disturbance speaker is a $51 / 4$-in woofer. Second, the dynamics of the duct interact with those of the speakers, and the strength of this interaction varies from mode to mode depending upon the relative locations of the speakers. Third, some modes may
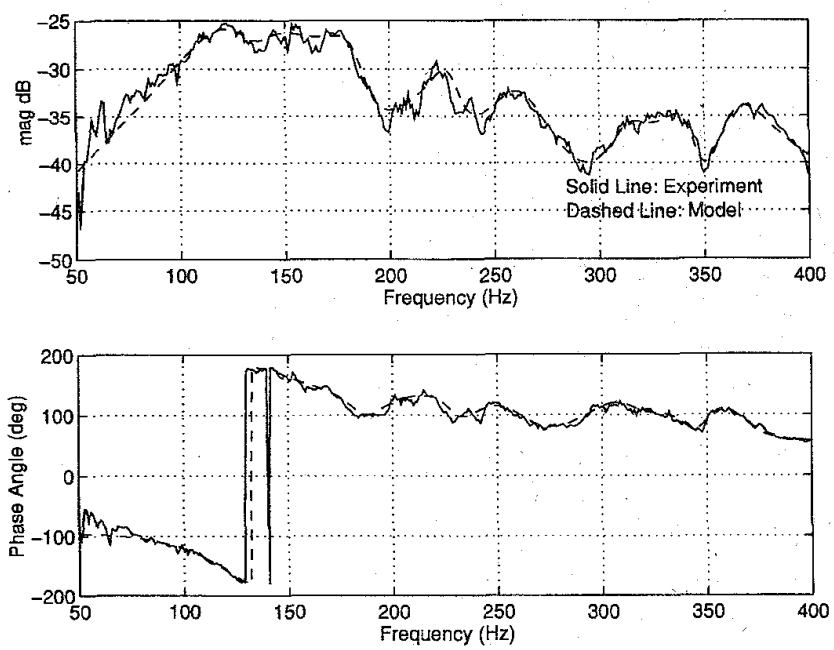

Fig. 5. Frequency response of the duct from control speaker to microphone.

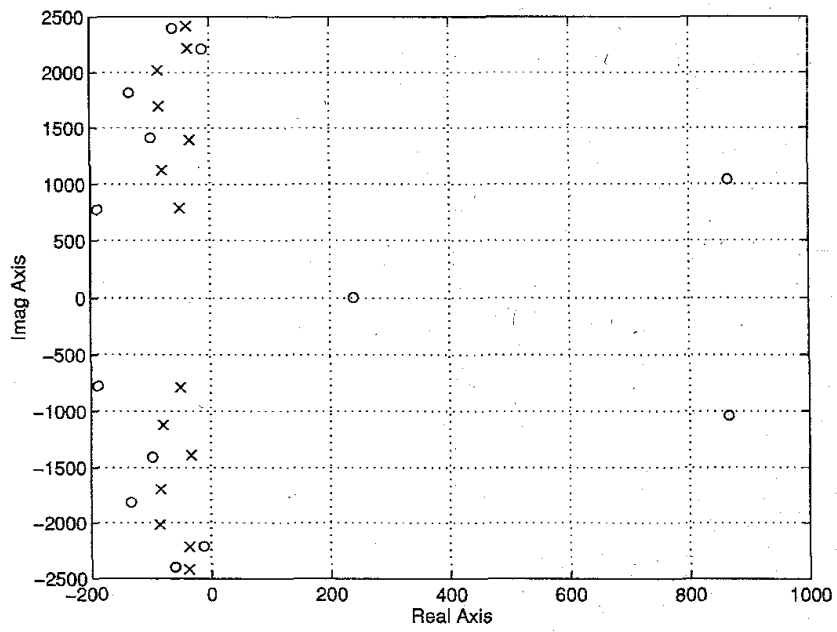

Fig. 6. Pole-zero map of the identified transfer function from disturbance speaker to microphone.

not be observable since the sensing microphone may be located at a node of those modes.

\section{FEEDBACK CONTROLlER DESIGN}

The control objective is to minimize the acoustic pressure at the performance point $x_{p}$. To obtain desired rolloff characteristics for robustness to high-frequency uncertainty, the precompensation technique described in [18] is used, and a precompensator $G_{p c}$ of order $n_{p c}$ is introduced as shown in Fig. 8. Note that precompensation is not a form of performance weighting, but rather a technique for influencing the loop shape. Let $G_{p c}$ have a realization

$$
\begin{aligned}
& \dot{x}_{p c}(t)=A_{p c} x_{p c}(t)+B_{p c} u(t) \\
& u_{p c}(t)=C_{p c} x_{p c}(t)
\end{aligned}
$$

This precompensator is cascaded with the plant of order $n_{\text {plant }}$ to obtain an augmented state-space representation of the plant 


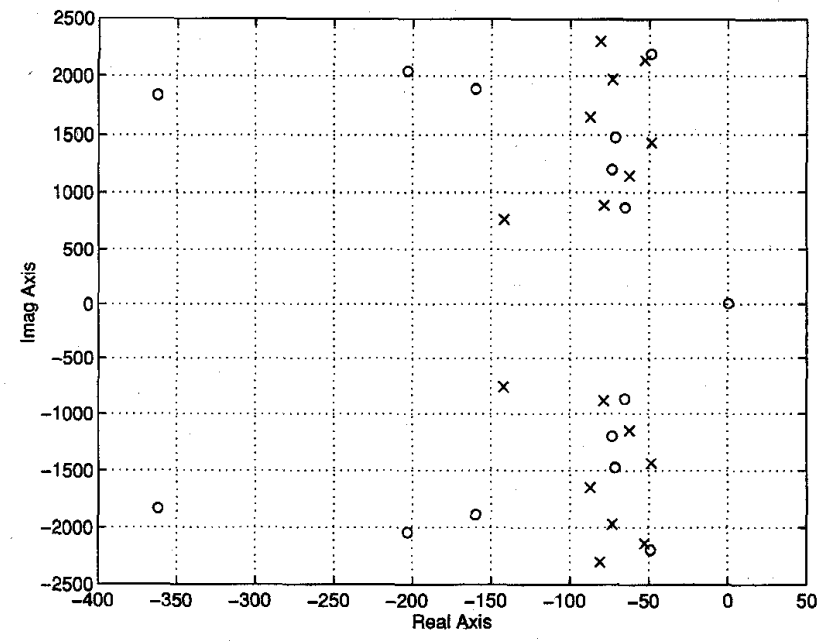

Fig. 7. Pole-zero map of the identified transfer function from control speaker to microphone.

and precompensator of order $\hat{n}=n_{\text {plant }}+n_{p c}$ given by

$$
\begin{aligned}
\dot{\hat{x}}(t) & =\hat{A} \hat{x}(t)+\hat{B} u(t)+\hat{D}_{1} w(t), \\
y(t) & =\hat{C} \hat{x}(t)+\hat{D}_{2} w(t), \\
z(t) & =\hat{E}_{1} \hat{x}(t)+\hat{E}_{2} u(t)
\end{aligned}
$$

where

$$
\begin{aligned}
\hat{x}(t) & \triangleq\left[\begin{array}{c}
x_{p c}(t) \\
x(t)
\end{array}\right], \\
\hat{A} & \triangleq\left[\begin{array}{cc}
A_{p c} & 0 \\
B C_{p c} & A
\end{array}\right], \\
\hat{B} & \triangleq\left[\begin{array}{c}
B_{p c} \\
0
\end{array}\right], \\
\hat{C} & \triangleq\left[\begin{array}{ll}
0 & C
\end{array}\right] \\
\hat{D_{1}} & \triangleq\left[\begin{array}{c}
0 \\
D_{1}
\end{array}\right] \\
\hat{D}_{2} & \triangleq D_{2} \\
\hat{E}_{1} & \triangleq\left[\begin{array}{ll}
0 & E_{1}
\end{array}\right] \\
\hat{E}_{2} & \triangleq E_{2} .
\end{aligned}
$$

For controller synthesis, the performance point is the sensing microphone location, and thus $E_{1}$ is taken to be equal to $C$. This choice obviates the need to calculate the matrix $E_{1}$ from the eigenfunctions, since $C$ is obtained directly from the identified transfer function. The control weighting matrix $E_{2}$ is chosen using trial and error, the object being to minimize control effort while obtaining good performance.

For the plant of order $n_{\text {plant }}$ described by (58), (59), and (62), we seek a controller $G_{c}$ with a realization of the form

$$
\begin{aligned}
\dot{x}_{c}(t) & =A_{c} x_{c}(t)+B_{c} y(t), \\
u(t) & =C_{c} x_{c}(t) .
\end{aligned}
$$

The first step in obtaining the above controller is to design a controller $\hat{G}_{c}$ of order $\hat{n}$ for the augmented plant of order $\hat{n}$

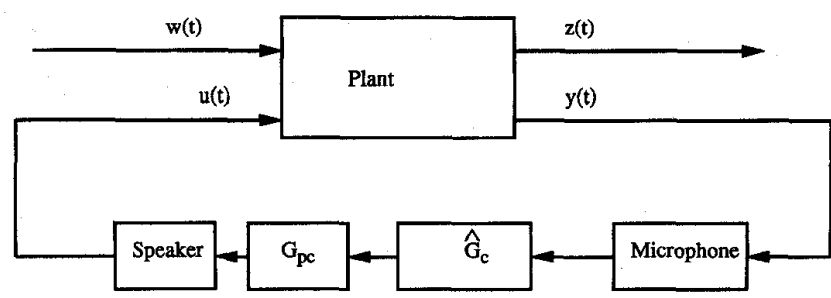

Fig. 8. Closed-loop configuration including precompensator.

described by (66)-(68). Let $\hat{G}_{c}$ have a realization

$$
\begin{aligned}
\dot{\hat{x}}_{c}(t) & =\hat{A}_{c} \hat{x}_{c}(t)+\hat{B}_{c} y(t), \\
u(t) & =\hat{C}_{c} \hat{x}_{c}(t) .
\end{aligned}
$$

Defining

$$
\tilde{x}(t) \triangleq\left[\begin{array}{c}
\hat{x}(t) \\
\hat{x}_{c}(t)
\end{array}\right]
$$

the closed-loop system becomes

$$
\begin{aligned}
& \dot{\tilde{x}}(t)=\tilde{A} \tilde{x}(t)+\tilde{D} w(t), \\
& \tilde{z}(t)=\tilde{E} \tilde{x}(t)
\end{aligned}
$$

where

$$
\begin{aligned}
\tilde{A} & \triangleq\left[\begin{array}{cc}
\hat{A} & \hat{B} \hat{C}_{c} \\
\hat{B}_{c} \hat{C} & \hat{A}_{c}
\end{array}\right], \\
\tilde{D} & \triangleq\left[\begin{array}{c}
\hat{D}_{1} \\
\hat{B}_{c} \hat{D}_{2}
\end{array}\right], \\
\tilde{E} & \triangleq\left[\begin{array}{ll}
\hat{E}_{1} & \hat{E}_{2} \hat{C}_{c}
\end{array}\right] .
\end{aligned}
$$

LQG synthesis minimizes the $\mathrm{H}_{2}$ norm of $\tilde{G}(s)$, the transfer function of the state-space realization (77), (78), and yields a controller of order $n_{p c}+n_{\text {plant }}$ given by

$$
\begin{aligned}
& \hat{A}_{c}=\hat{A}+\hat{B} \hat{C}_{c}-\hat{B}_{c} \hat{C}, \\
& \hat{B}_{c}=\left(Q \hat{C}^{T}+V_{12}\right) V_{2}^{-1} \\
& \hat{C}_{c}=-R_{2}^{-1}\left(\hat{B}^{T} P+R_{12}^{T}\right)
\end{aligned}
$$

where $V_{2} \triangleq \hat{D}_{2} \hat{D}_{2}^{T}, \quad V_{12} \triangleq \hat{D}_{1} \hat{D}_{2}^{T}, R_{2} \triangleq \hat{E}_{2}^{T} \hat{E}_{2}$, $R_{12} \triangleq \hat{E}_{1}^{T} \hat{E}_{2}$, and $Q$ and $P$ satisfy the Riccati equations

$$
\begin{aligned}
\hat{A} Q & +Q \hat{A}^{T}+V_{1} \\
& -\left(Q \hat{C}^{T}+V_{12}\right) V_{2}^{-1}\left(Q \hat{C}^{T}+V_{12}\right)^{T}=0, \\
\hat{A}^{T} P & +P \hat{A}+R_{1} \\
& -\left(P \hat{B}+R_{12}\right) R_{2}^{-1}\left(P \hat{B}+R_{12}\right)^{T}=0 .
\end{aligned}
$$

The implemented controller $G_{C}$ given in (72) and (73) is now obtained by cascading the precompensator $G_{p c}$ with the controller $\hat{G}_{c}$ described by (82). This yields the controller

$$
\begin{aligned}
A_{c} & =\left[\begin{array}{cc}
\hat{A}_{c} & 0 \\
B_{p c} \hat{C}_{c} & A_{p c}
\end{array}\right], \\
B_{c} & =\left[\begin{array}{c}
\hat{B}_{c} \\
0
\end{array}\right], \\
C_{c} & =\left[\begin{array}{ll}
0 & C_{p c}
\end{array}\right]
\end{aligned}
$$



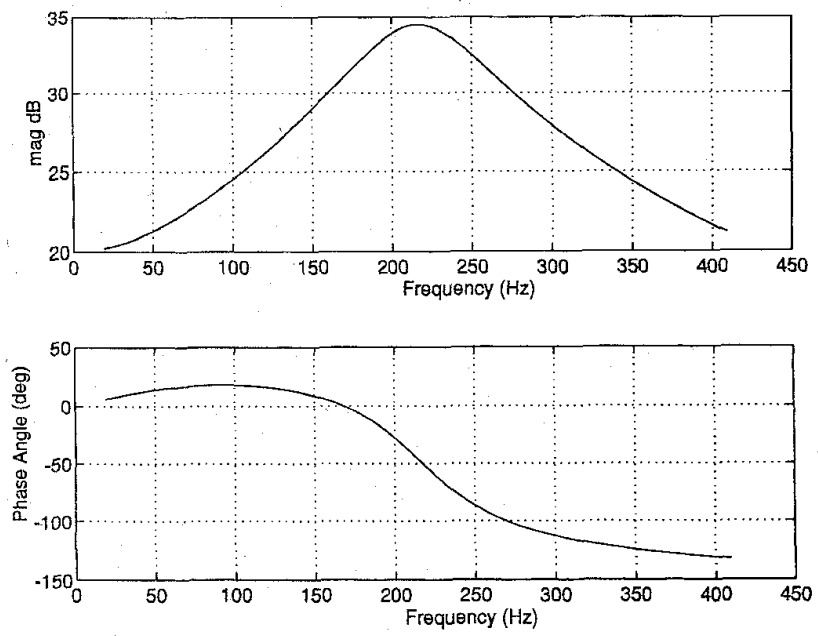

Fig. 9. Frequency response of the precompensator $G_{p c}$.
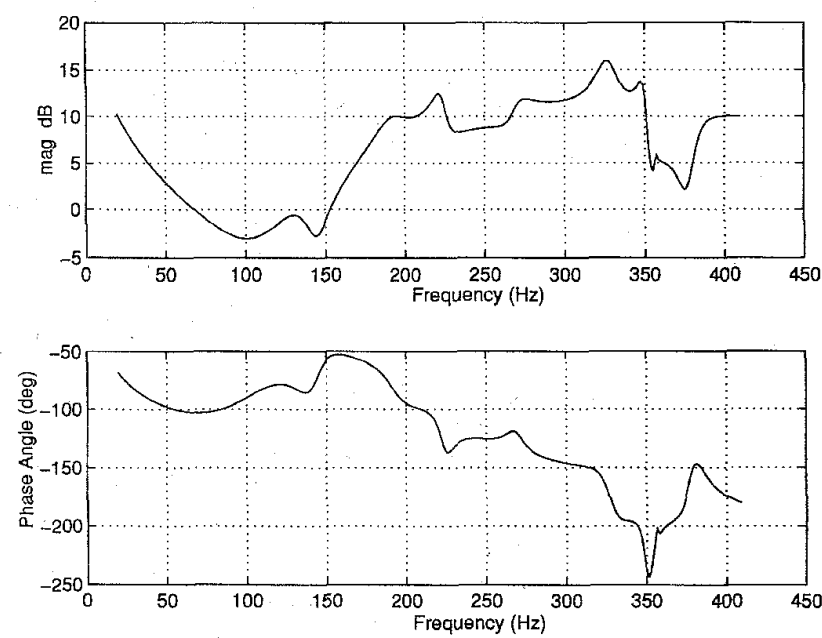

Fig. 10. Frequency response of the reduced-order controller $G_{c, r e d}$.

which is of order $n_{c}=n_{\text {plant }}+2 n_{p c}$. The controller is discretized and implemented digitally using a dSPACE realtime control interface board. The discrete-time controller has a realization given by

$$
\begin{aligned}
& A_{c, h}=e^{A_{c} h} \\
& B_{c, h}=H(h) B_{c}, \\
& C_{c, h}=\frac{1}{h} C_{c} H(h)
\end{aligned}
$$

where $H(s) \triangleq \int_{0}^{s} e^{A_{c} r} d r$ and $h=0.0002 \mathrm{~s}$ is the sampling interval corresponding to a $5-\mathrm{kHz}$ sample rate.

For a precompensator of order $n_{p c}=3$, the resulting controller $G_{c}$ is of order $n_{c}=36$. By applying standard balancing and truncation techniques, a controller $G_{c, \text { red }}$ of order $n_{c, \text { red }}=22$ is obtained with little loss of performance. Frequency response plots of the precompensator, the reducedorder controller, the loop gain, and open-loop (no control) and closed-loop performance at the sensor location and at the end of the duct are shown in Figs. 9-13. From Fig. 12 it can be
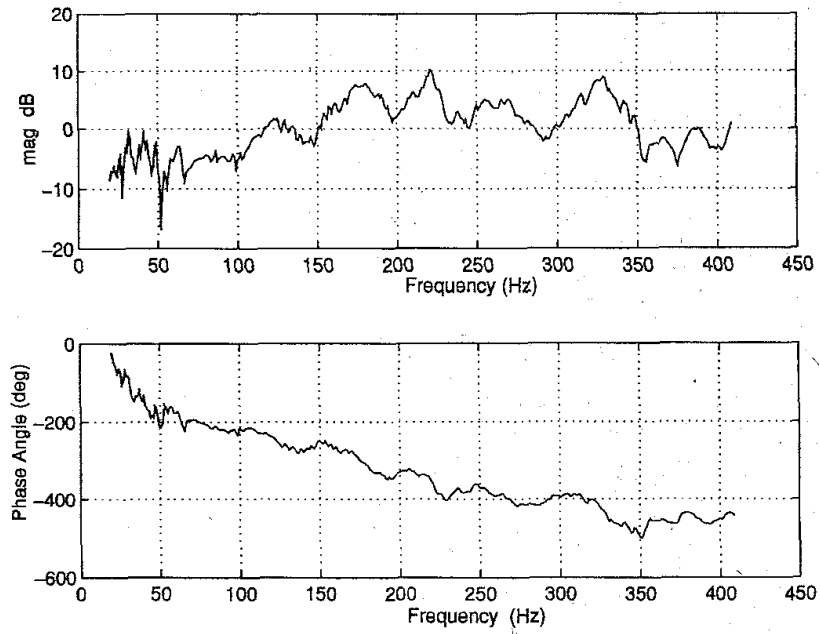

Fig. 11. Frequency response of the loop gain.
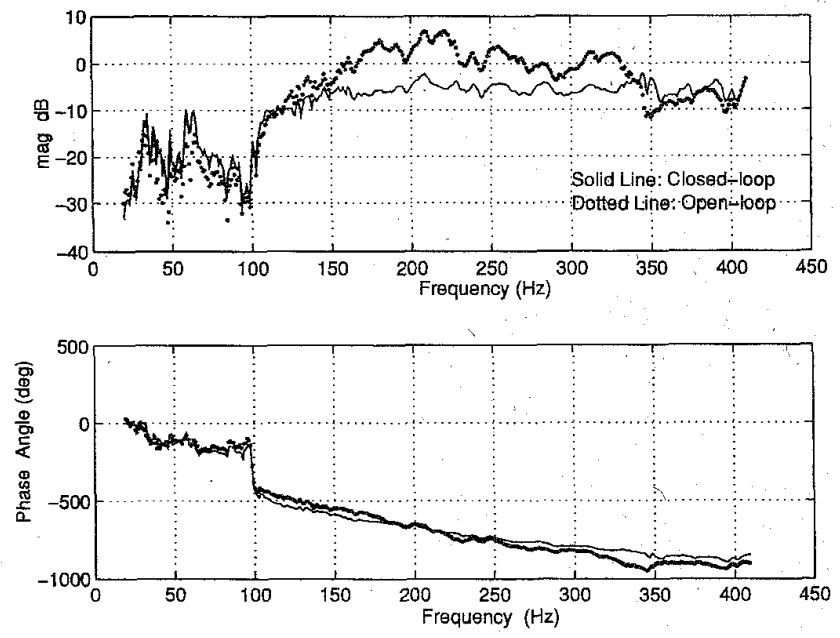

Fig. 12. Open-loop and closed-loop performance at $x_{m}$.

seen that noise reduction of $5 \mathrm{~dB}$ to $12 \mathrm{~dB}$ is obtained at the sensor location $x_{m}$ over a bandwidth of about $200 \mathrm{~Hz}$ using the control techniques described above. Fig. 13 shows the open-loop and closed-loop performances at the end of the duct away from the disturbance source. It can be seen that significant noise attenuation is obtained over a bandwidth of about $200 \mathrm{~Hz}$. The performance plots show the magnitude and phase of the microphone output voltage; the reduction in sound pressure level (SPL) is not known. SPL data, however, are not currently available to compare the performance to other acoustic control schemes.

\section{DISCUSSION AND CONCLUSIONS}

Reduction in noise levels in the acoustic duct is obtained using precompensated LQG feedback controller synthesis techniques. The experimental identification of the duct provides the numerical parameters used for controller synthesis. The closed-loop performance shows a significant reduction in noise level over a reasonable bandwidth with a simple control 


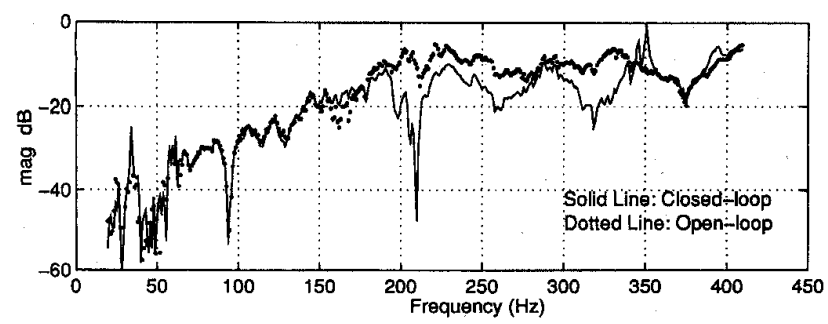

Fig. 13. Open-loop and closed-loop performance at the end of the duct.

design procedure. Using this design procedure, it is found that much higher levels of noise attenuation can be obtained below $400 \mathrm{~Hz}$ at the expense of creating peaks at frequencies beyond the model bandwidth. As shown in [20], this spillover phenomenon is a direct result of the Bode integral constraint on sensitivity. More sophisticated control schemes involving multiple sensors and actuators are to be investigated. Finally, direct robust reduced-order controller synthesis techniques remain to be applied to obtain lower-order controllers with specified gain and phase margins.

\section{ACKNOWLEDGMENT}

The authors thank Prof. C. Radcliffe for valuable guidance and suggestions and Dr. C. Nett for helpful suggestions.

\section{REFERENCES}

[1] P. Lueg, "Process of silencing sound oscillations," U.S. Patent 2043416 , 1936.

[2] H. F. Olson and E. G. May, "Electronic sound absorber," J. Acoust. Soc. Amer., vol. 25, pp. 1130-1136, 1953.

[3] P. A. Nelson and S. J. Elliot, Active Control of Sound. New York: Academic, 1992.

[4] S. J. Elliot, I. M. Stothers, and P. A. Nelson, "A multiple error LMS algorithm and its applications to the active control of sound and vibration," IEEE Trans. Acoust., Speech, Signal Processing, vol ASSP-35, pp. 1423-1434, 1987.
[5] C. R. Fuller, C. A. Rogers, and H. H. Robertshaw, "Control of sound radiation with active/adaptive structures," J. Sound Vibration, vol. 157, pp. 19-39, 1992.

[6] A. J. Hull, C. J. Radcliffe, M. Miklavcic, and C.. R. MacCluer, "Statespace representation of the nonself-adjoint acoustic duct system," $J$. Vibration Acoust., vol. 112, pp. 483-488, 1990.

[7] C. J. Radcliffe and S. D. Gogate, "Identification and modeling of speaker dynamics for acoustic control applications," in Active Control of Noise and Vibration. Amer. Soc. Mech. Eng., book G00737, vol. 38, pp. 295-300, 1992.

[8] A. J. Hull, C. J. Radcliffe, and S. C. Southward, "Global active noise control of a one-dimensional acoustic duct using a feedback controller," J. Dynamic Syst., Measurement, Contr., vol. 115, pp. 488-494, 1993.

[9] X. H. Yang, J. van Niekerk, K. S. Parwani, A. Packard, and J. Tingue, "Attenuation of structurally generated interior noise through active control," in Proc. Amer. Contr. Conf., San Francisco, CA, 1993, pp. $1-7$.

[10] B. Hassibi, A. H. Sayed, and T. Kailath, "LMS is $H^{\infty}$ optimal," in Proc. IEEE Conf. Decision Contr., San Antonio, TX, 1993, pp. 74-79.

[11] D. G. MacMartin, "A feedback perspective on the LMS disturbance feedforward algorithm," in Proc. Amer. Contr. Conf., Baltimore, MD, 1994, pp. 1632-1636.

[12] A. D. Pierce, Acoustics: An Introduction to Its Physical Principles and Applications. New York: Acoust. Soc. Amer., 1991.

[13] P. E. Doak, "Excitation, transmission, and radiation of sound from source distribution in hard-walled ducts of finite length," $J$. Sound Vibration, vol. 31, pp. 1-72, 1973.

[14] L. E. Kinsler, A. R. Frey, A. B. Collins, and J. V. Saunders, Fundamentals of Acoustics. New York: Wiley, 1982

[15] W. K. W. Hong, K. Egthesadi, and H. G. Leventhall, "The tandem tight-coupled active noise attenuator in a duct," Acoust. Lett., vol, 6, pp. 19-24, 1982.

[16] K. Eghtesadi, W. K. W. Hong, and H. G. Leventhall, "The tight-coupled monopole active attenuator in a duct," Noise Contr. Eng. J., vol. 20, pp. $16-20,1983$

[17] W. K. W. Hong, K. Eghtesadi, and H. G. Leventhall, "The tight-coupled monopole (TCM) and the tight-coupled tandem (TCT) attenuators: Theoretical aspects and experimental attenuation in an air duct," $J$. Acoust. Soc. Amer., vol. 81, pp. 376-388, 1987.

[18] E. G. Collins, J. A. King, and D. S. Bernstein, "Application of maximum entropy/optimal projection design synthesis to a benchmark problem," J. Guidance, Contr., Dynamics, vol. 15, pp. 1094-1102, 1992.

[19] J. Hong and D. S. Bernstein, "A comparison of the fundamental properties of feedback and feedforward control: Bode constraints and spillover," in Proc. Active '95, Newport Beach, CA, July 1995. 